\title{
AVALIAÇÃO FINANCEIRA DE PLANTAÇÕES DE EUCALIPTO SUBMETIDAS A DESBASTE $^{1}$
}

Andrea Nogueira Dias², Helio Garcia Leite ${ }^{3}$, Márcio Lopes da Silva $^{3}$ e Andrelino Ferreira de Carvalho ${ }^{4}$

RESUMO - O objetivo deste estudo foi determinar o regime de manejo mais rentável, dentre as variações de idade e intensidades de desbaste, índice de local, taxas de juros, preços de madeira e idades de corte final. Para tanto, utilizaram-se dados de um experimento de desbaste instalado na Empresa Copener Florestal Ltda., Bahia. O sistema de prognose empregado foi desenvolvido por Clutter (1963). O método de avaliação financeira utilizado foi o Benefício Periódico Equivalente (BPE). Os resultados indicaram que o regime com desbaste foi mais rentável economicamente, comparado ao regime sem desbaste. No entanto, à medida que aumentou a intensidade de desbaste, os valores de BPE tenderam a diminuir. Pôde-se observar, também, que menores taxas de juros e maiores índices de local tenderam a aumentar a rentabilidade dos regimes. O regime que proporcionou maior rentabilidade foi o do desbaste realizado aos 4 anos e o corte final aos 9 anos. Não houve influência significativa das variáveis índice de local, taxa de juros e custo de implantação, na determinação do regime mais rentável, ou seja, o regime mais rentável permaneceu sendo do desbaste aos 4 e corte final aos 9 anos. No entanto, a variável preço da madeira do desbaste influenciou a determinação do regime mais rentável, e o aumento no preço de acordo com a idade de colheita resultou em desbaste aos 6 anos e o corte final aos 9 anos, como o regime mais rentável. Da mesma forma, a elevação no preço da madeira do corte final, de acordo com a idade de corte, resultou em desbaste aos 4 anos e corte final aos 12 anos, como o regime mais rentável. A simulação de elevação no preço da madeira do desbaste e do corte final, simultaneamente ao mesmo tempo, gerou como regime de manejo mais rentável o desbaste aos 6 anos e o corte final aos 12 anos. Concluiuse que houve influência direta das variáveis preço da madeira do desbaste e do corte final na determinação do regime mais rentável.

Palavras-chave: Benefício periódico equivalente, desbaste, simulação e eucalipto.

\section{ECONOMIC EVALUATION OF EUCALYPTUS PLANTATION SUBMITTED TO THINNING}

\begin{abstract}
The objective of this work was to determine the most profitable management method, among the variation of age and thinning intensities, site index, interest rates, wood prices and last cutting age. To achieve this goal, data of a thinning experiment established at Empresa Copener Florestal Ltda, Bahia, were used. The prediction system used was developed by Clutter (1963). The economic evaluation method used was the Equivalent Periodic Benefit (BPE). The results showed that the method with thinning was economically better than the method without thinning, but as the thinning intensity increased the BPE values tended to
\end{abstract}

\footnotetext{
${ }^{1}$ Recebido em $1^{\circ}$ 09.2003 e aceito para publicação em 20.04.2005.

${ }^{2}$ Departamento de Engenharia Florestal da Unicentro, Irati-PR. E-mail: <andias@ irati.unicentro.br>.

${ }^{3}$ Departamento de Engenharia Florestal da UFV. Viçosa-MG. E-mail: <marlosil@ mail.ufv.br $>,<$ hgleite@ mail.ufv.br>.

${ }^{4}$ COPENER FLORESTAL, BA.
} 
decrease. Also, lower interest rates and higher site indexes were observed to increase the method profits. The method that provided the greatest profit was obtained with thinning at four and last cut at nine years of age. The variables site index, interest rate and establishment cost did not significantly influence the determination of the most profitable method, that is, the most profitable method remained the thinning at four and last cut at nine years of age. On the other hand, the variable thinned wood price influenced the determination of the most profitable method. The increase in price according to the harvesting age resulted in thinning at six and last cut at nine years of age as the most profitable method. Similarly, the increase in wood price of the last cut according to the cutting age resulted in thinning at four and last cut at 12 years of age as the most profitable method. The simulation of increasing the price of thinned and last cut wood, at the same time, generated as the most profitable method thinning at six and last cut at 12 years of age. It was concluded that there was a direct influence of variables prices of thinned and last cut wood on the determination of the most profitable method.

Key words: Equivalent periodic benefit, thinning, simulation and eucalypt.

\section{INTRODUÇÃO}

Nas últimas décadas, as empresas do setor florestal têm buscado aprimorar a produção de madeira, visando atender ao mercado consumidor, que tem exigido produtos de base florestal de maior valor agregado. Com isso, é imprescindível a adoção de novas formas de manejar os povoamentos, incluindo a técnica de desbastar os povoamentos, para eliminar indivíduos inferiores e disponibilizar luz, água e nutrientes àqueles considerados superiores, tudo isso com o intuito de buscar, prioritariamente, a qualidade do produto final e, assim, aumentar a rentabilidade do investimento.

As plantações de eucalipto no Brasil tradicionalmente foram manejadas sem a aplicação de desbastes. A carência de pesquisas que norteiem as operações de desbastes tem impedido a definição adequada quanto à escolha e idade das árvores a serem desbastadas, bem como a escolha da intensidade e do número de desbastes para cada local e espécie. Além disso, as informações sobre a taxa de retorno a ser obtida com a adoção dos desbastes em florestas de eucalipto são escassas (BERTOLOTTI et al., 1983).

Os efeitos dos desbastes no povoamento estão relacionados ao tipo, intervalo e intensidade do desbaste, e para analisar esses efeitos corretamente é necessário adicionar, aos dados de produção, informações de caráter econômico do povoamento (CLUTTER et al., 1983).

O objetivo principal deste trabalho foi determinar o regime de manejo mais rentável economicamente em povoamentos de eucalipto desbastados.

R. Árvore, Viçosa-MG, v.29, n.3, p.419-429, 2005
Dentre os objetivos específicos, citam-se: 1) Comparar os regimes de manejo, considerando o desbaste e sem considerar a aplicação do desbaste, em termos econômicos; e 2) Avaliar a influência das variáveis índice de local, taxa de juros, custo de implantação e preço da madeira sobre a determinação do regime mais rentável economicamente.

\section{MATERIAL E MÉTODOS}

\subsection{Dados}

Utilizou-se uma base de dados proveniente do estudo realizado por Dias (2000) na empresa COPENER FLORESTAL Ltda., localizada na região nordeste da Bahia.

\subsection{Sistema de prognose do crescimento e da produção}

O sistema de equações desenvolvido por Clutter (1963) e ajustado por Dias (2000) foi utilizado para predizer o crescimento e a produção em volume e área basal:

- Produção futura em volume:

$\operatorname{Ln} \hat{V}_{2}=2,441896-24,23312 / I_{2}+0,032370 S+0,726114 \operatorname{LnB}_{2}$ $\mathrm{R}^{2}=0,73 \quad$ Bias $=-19,3825$

- Produção futura em área basal:

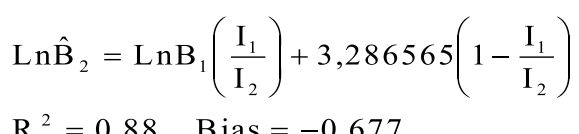
em que:

$\mathrm{V}_{2}=$ volume, em $\mathrm{m}^{3} / \mathrm{ha}$, na idade $\mathrm{I}_{2}$; 
$\mathrm{B}_{1}$ = área basal, em $\mathrm{m}^{2} / \mathrm{ha}$, na idade $\mathrm{I}_{1}$;

$\mathrm{B}_{2}=$ área basal, em $\mathrm{m}^{2} / \mathrm{ha}$;

$\mathrm{I}_{1=}$ Idade inicial de projeção, em meses;

$I_{2}=$ Idade posterior $\mathrm{I}_{1}$, em meses;

$\mathrm{S}=$ índice de local, em $\mathrm{m}$; e

$\mathrm{Ln}=\log$ aritmo neperiano.

\subsection{Método de avaliação financeira utilizado}

O Benefício Periódico Equivalente (BPE), critério indicado na seleção de projetos que apresentam durações ou vidas úteis diferentes, foi utilizado neste trabalho. O critério do BPE converte os fluxos de caixa líquidos em uma série equivalente de valores iguais e, geralmente, anuais (REZENDE e OLIVEIRA, 1995).

O BPE para cada projeto foi obtido por meio da fórmula usual:

$$
\begin{aligned}
& B P E=\frac{V P L\left[(1+i)^{t}-1\right]}{\left[1-\frac{1}{(1+i)^{n t}}\right]}, \text { sendo } \\
& \mathrm{VPL}=\sum_{\mathrm{j}=1}^{\mathrm{n}} \mathrm{R}_{\mathrm{j}}(1+\mathrm{i})^{-\mathrm{j}}-\sum_{\mathrm{j}=1}^{\mathrm{n}} \mathrm{C}_{\mathrm{j}}(1+\mathrm{i})^{-\mathrm{j}}
\end{aligned}
$$

em que:

$\mathrm{VPL}=$ valor presente líquido, em US\$/ha;

$\mathrm{Rj}=$ receita no período $\mathrm{j}$;

$\mathrm{Cj}=$ custo no período $\mathrm{j}$;

$\mathrm{i}=$ taxa de desconto;

$\mathrm{j}$ = período de ocorrência dos custos e receitas;

$\mathrm{n}=$ duração do projeto, em anos; e

$\mathrm{t}=\mathrm{n}^{\circ}$ de períodos de capitalização por ano.

\subsection{Sistema de simulação de regimes de manejo}

Os valores de produção em volume e área basal foram obtidos a partir da tabela de produção de densidade variável (Quadro 1). Para obter o volume desbastado e o volume do corte final, utilizados no fluxo de caixa, foram feitas simulações de desbastes, em \% de redução em área basal, na tabela de produção. Esses volumes foram utilizados posteriormente no fluxo de caixa, para determinação dos valores de BPE para cada projeto.

Denominou-se como um projeto cada cenário construído, de acordo com as simulações de idade de desbaste e de corte final, de intensidades de desbaste, de índice de local, de área basal inicial (24 meses), de taxas de juros, dos custos de colheita e do preço da madeira do desbaste e do corte final (Figura 1).

Quadro 1 - Tabela de produção de densidade variável para o híbrido Eucalyptus grandis X E.urophylla, no município de Alagoinhas, Bahia

Table 1 - Variable density yield table for the Eucalyptus grandis X E.urophylla hybrid, in the municipality of Alagoinhas,

\begin{tabular}{|c|c|c|c|c|c|c|c|c|c|}
\hline \multirow[b]{3}{*}{$\begin{array}{c}\text { Idade } \\
\text { (meses) }\end{array}$} & \multicolumn{9}{|c|}{ Índice de Local* ( idade- índice de 50 meses) } \\
\hline & \multicolumn{3}{|c|}{17} & \multicolumn{3}{|c|}{21} & \multicolumn{3}{|c|}{25} \\
\hline & $\begin{array}{c}\text { Á. Basal } \\
\left(\mathrm{m}^{2} / \mathrm{ha}\right)\end{array}$ & $\begin{array}{l}\text { Volume } \\
\left(\mathrm{m}^{3} / \mathrm{ha}\right)\end{array}$ & $\begin{array}{c}\text { IMM } \\
\left(\mathrm{m}^{2} / \mathrm{ha}\right)\end{array}$ & $\begin{array}{c}\text { Á. Basal } \\
\left(\mathrm{m}^{3} / \mathrm{ha}\right)\end{array}$ & $\begin{array}{l}\text { Volume } \\
\left(\mathrm{m}^{2} / \mathrm{ha}\right)\end{array}$ & $\begin{array}{c}\text { IMM } \\
\left(\mathrm{m}^{3} / \mathrm{ha}\right)\end{array}$ & Á. Basal & Volume & IMM \\
\hline 24 & 7,50 & 31,36 & 1,307 & 8,50 & 39,09 & 1,629 & 9,00 & 46,38 & 1,932 \\
\hline 30 & 9,67 & 46,16 & 1,539 & 10,69 & 56,5 & 1,883 & 11,19 & 66,49 & 2,216 \\
\hline 36 & 11,46 & 59,73 & 1,659 & 12,46 & 72,24 & 2,007 & 12,94 & 84,53 & 2,348 \\
\hline 42 & 12,93 & 71,81 & 1,710 & 13,89 & 86,09 & 2,050 & 14,35 & 100,34 & 2,389 \\
\hline 48 & 14,16 & 82,44 & 1,717 & 15,08 & 98,2 & 2,046 & 15,52 & 114,12 & 2,377 \\
\hline 54 & 15,2 & 91,79 & 1,700 & 16,07 & 108,78 & 2,014 & 16,48 & 126,12 & 2,336 \\
\hline 60 & 16,09 & 100,02 & 1,667 & 16,91 & 118,06 & 1,968 & 17,3 & 136,63 & 2,277 \\
\hline 66 & 16,85 & 107,30 & 1,626 & 17,63 & 126,24 & 1,913 & 18,00 & 145,88 & 2,210 \\
\hline 72 & 17,51 & 113,78 & 1,580 & 18,25 & 133,49 & 1,854 & 18,61 & 154,06 & 2,140 \\
\hline 78 & 18,09 & 119,56 & 1,533 & 18,80 & 139,95 & 1,794 & 19,13 & 161,34 & 2,068 \\
\hline 80 & 18,27 & 121,35 & 1,517 & 18,97 & 141,94 & 1,774 & 19,29 & 163,59 & 2,045 \\
\hline 82 & 18,44 & 123,08 & 1,501 & 19,13 & 143,87 & 1,755 & 19,45 & 165,76 & 2,021 \\
\hline
\end{tabular}
Bahia

Fonte: Dias (2000) - IMM = incremento médio mensal ( $\left.\mathrm{m}^{3} / \mathrm{ha} / \mathrm{ano}\right)$.

*Índice de local $=$ representa a altura dominante média do povoamento na idade-índice estabelecida (neste caso, de 50 meses). 


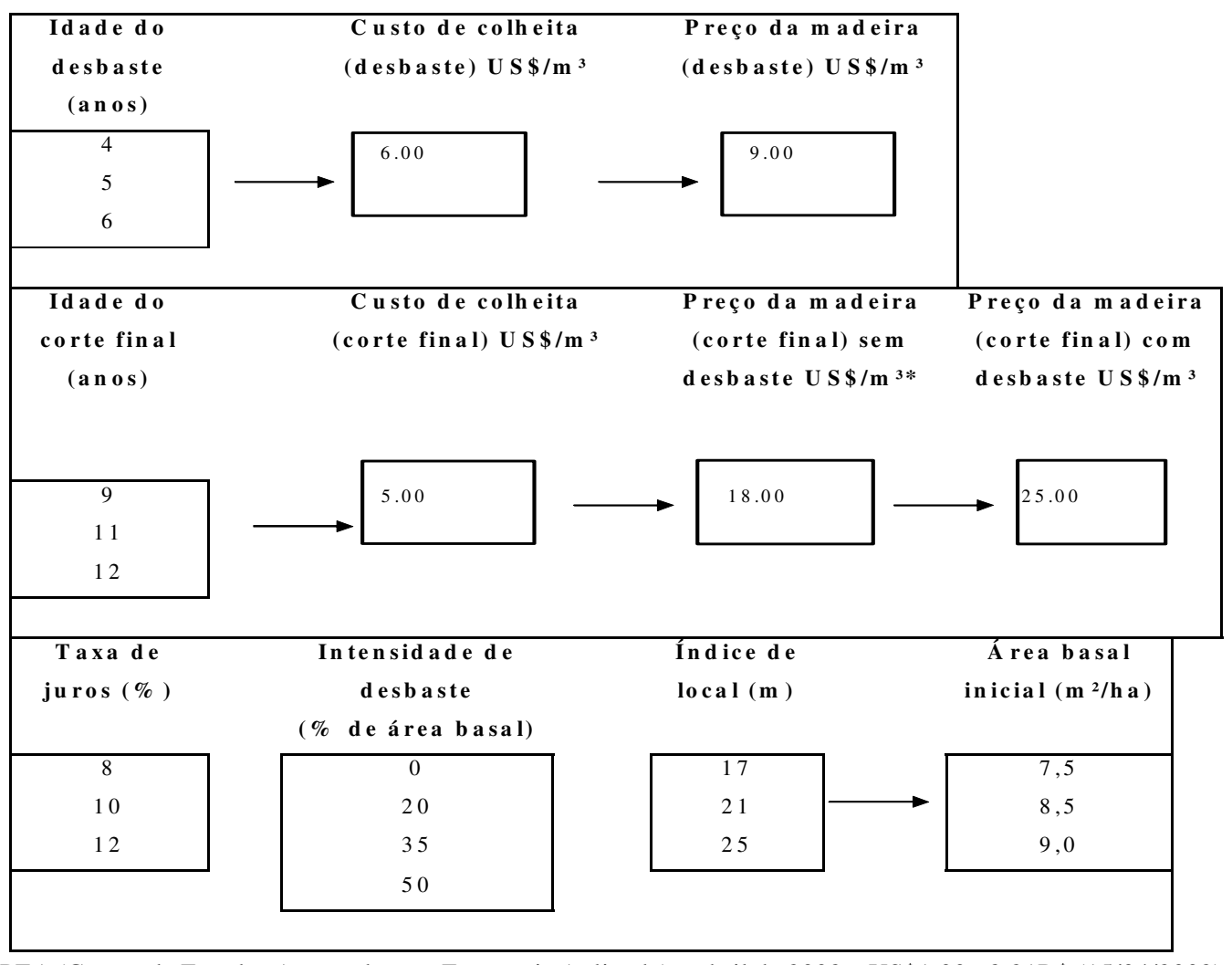

*Fonte: CEPEA (Centro de Estudos Avançados em Economia Aplicada) - abril de 2003 - US \$1,00=3,21R \$ (15/04/2003).

Figura 1 - Cenários de custos, preços, índice de local, área basal e taxa de juros .

Figure 1 -Scenario of costs, prices, site index, basal area and interest rates.

No fluxo de caixa, utilizaram-se informações de custos médios de condução de povoamentos de eucalipto aplicados pelas empresas florestais e as receitas obtidas para cada projeto (Tabela 1). As receitas se referem ao valor do volume de madeira de acordo com o preço atribuído na ocasião do desbaste e do corte final, enquanto os preços, ao volume de madeira por $\mathrm{m}^{3}$.

Tabela 1 - Dados de custos de produção de madeira para cada atividade

Table 1 - Data on costs of wood production per activity

\begin{tabular}{lcc}
\hline Atividade & Ano de Ocorrência & Custo (US\$/ha) \\
\hline Implantação & 0 & 400.00 \\
Capina 1 & 1 & 50.00 \\
Capina 2 & 2 & 20.00 \\
Manutenção & Anual & 10.00 \\
Terra & Anual & $(350.00 \times$ Taxa de juros) \\
Administração & Anual & $10 \%$ do custo total \\
\hline US $\$ 1.00=3,21 \mathrm{R} \$(15 / 04 / 2003)$.
\end{tabular}

US\$1.00=3,21R $\$(15 / 04 / 2003)$

R. Árvore, Viçosa-MG, v.29, n.3, p.419-429, 2005

\subsection{Análise da influência de variáveis na determinação da idade de desbaste e do corte final}

Com o objetivo de analisar a influência de algumas variáveis na determinação do regime de manejo mais rentável, obtiveram-se os valores de BPE de cada projeto, considerando simulações no fluxo de caixa, de desbaste aos 4, 5 e 6 anos, de corte final aos 9, 11 e 12 anos, de índice de local $(17,21$ e $25 \mathrm{~m})$, de taxa de juros $(8$, 10 e $12 \%$ ) e de custo de implantação.

Para a variável preço da madeira do desbaste, considerou-se uma elevação do preço da madeira com a idade de colheita, devido a uma melhor utilização da madeira originária do desbaste em decorrência da idade de corte. Para a variável preço da madeira do corte final, considerou-se uma elevação no preço da madeira do corte final, em razão da melhoria da qualidade da madeira e produção de árvores de maior valor individual após o desbaste. 
Assim, os valores de BPE foram obtidos da mesma forma, porém, para analisar a influência dessas variáveis, utilizaram-se às variações descritas na Figura 2.

\section{RESULTADOS E DISCUSSÃO}

\subsection{Análise econômica dos regimes de manejo}

\subsubsection{Intensidade de desbaste}

Para esta análise, obtiveram-se os valores de BPE para cada regime de manejo. Como exemplo, consideraram-se para um índice de local de $25 \mathrm{~m}$ área basal inicial aos 24 meses de $9,0 \mathrm{~m}^{2} /$ ha e a taxa de juros de $8 \%$ ao ano, variações de idade de desbaste $(4,5$ e 6 anos), intensidade de desbaste $(0,20,35$ e $50 \%)$ e idade de corte final ( 9,11 e 12 anos).

Os custos e preços de madeira utilizados estão descritos na Tabela 1 e Figura 1, respectivamente. Para o regime com desbaste, considerou-se um preço de madeira superior em $40 \%$ ao do regime sem desbaste (Figura 1). Estes valores serão adotados nas análises dos itens 3.1.2 e 3.1.3.

Os resultados indicaram que, apesar de os valores de BPE se apresentarem positivos em qualquer uma das situações testadas, o regime sem desbaste exibiu menores valores de BPE. Para os regimes que consideraram a ocorrência de desbaste, notou-se que o aumento na intensidade de desbaste ocasionou decréscimos dos valores de BPE, para qualquer idade de desbaste e corte final testada (Quadro 2).

Verificou-se que não houve diferença nos valores de BPE obtidos nos regimes de manejo que utilizaram as simulações de desbaste aos 4, 5 ou 6 anos. Porém, os maiores valores de BPE foram obtidos na simulação de desbaste aos quatro anos, para qualquer intensidade analisada (Quadro 2). Verificou-se também que, independentemente de a idade de desbaste ser 4, 5 ou 6 anos, os maiores valores de BPE ocorreram nas simulações de corte final aos 9 anos, em qualquer intensidade (Figura 3). Nessa simulação, a idade de desbaste considerada foi de 4 anos, índice de local de 25 e taxa de juros de $8 \%$ ao ano.

\subsubsection{Taxa de juros}

Considerou-se, neste caso, a realização do desbaste aos 4 anos e do corte final aos 9 anos para o índice de local de $25 \mathrm{~m}$ e as taxas de juros de 8, 10 e $12 \%$ ao ano. Observou-se na Figura 4, conforme o esperado, que maiores taxas de juros resultaram em redução dos benefícios, medidos em BPE (US\$.ha-1 $\mathrm{ano}^{-1}$ ).

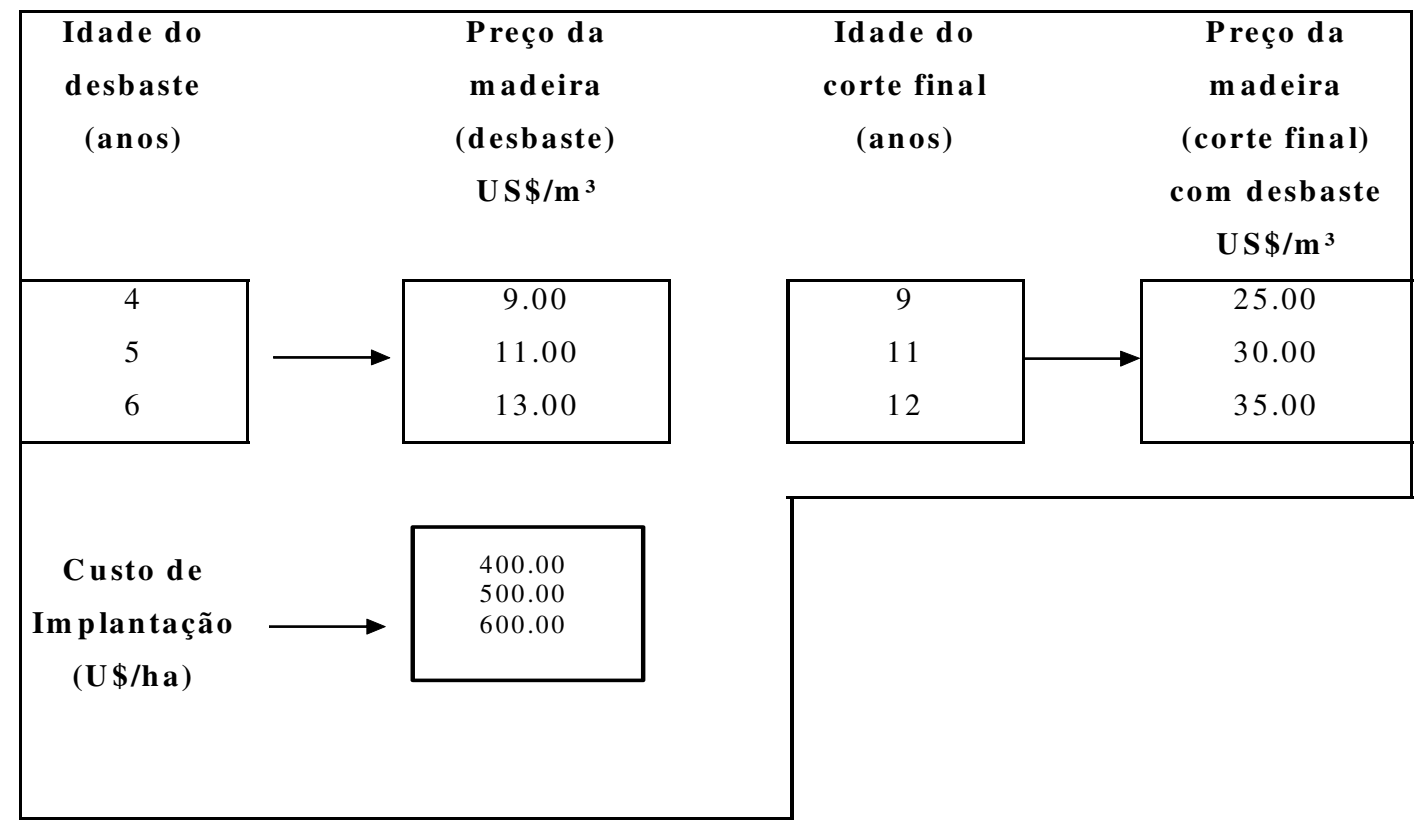

Figura 2 - Variações de preço da madeira de acordo com a idade de desbaste e do corte final e de custos de implantação. Figure 2 - Variations on wood prices according to thinning age, last cutting age and costs of stand establishment. 
Quadro 2 - BPE (em US\$.ha ${ }^{-1} \cdot$ ano $^{-1}$ ) para simulação de desbaste aos 4, 5 e 6 anos, com 0, 20, 35 e $50 \%$ de intensidade, para o índice de local $25 \mathrm{~m}$, taxa de juros de $8 \%$ e corte final aos 9,11 e 12 anos

Table $2-B P E\left(\right.$ in US\$.h $a^{-1}$.year ${ }^{-1}$ ) for thinning simulation at four, five and six years of age, with $0,20,35$ and $50 \%$ intensity, for the site index of $25 \mathrm{~m}$, interest rate of $8 \%$ and last cut at nine, 11 and 12 years of age

\begin{tabular}{|c|c|c|c|c|}
\hline Desbaste (\%) & $\begin{array}{c}\text { Id Corte Final } \\
(\text { anos })\end{array}$ & $\begin{array}{c}* 4-\mathrm{BPE} \\
\left(\mathrm{US} \$ . \mathrm{ha}^{-1} \mathrm{ano}^{-1}\right)\end{array}$ & $\begin{array}{c}5-\mathrm{BPE} \\
\left(\mathrm{US} \$ . \mathrm{ha}^{-1} \mathrm{ano}^{-1}\right)\end{array}$ & $\begin{array}{c}6-\mathrm{BPE} \\
\left(\mathrm{US} \$ \cdot \mathrm{ha}^{-1} \mathrm{ano}^{-1}\right)\end{array}$ \\
\hline 0 & 9 & 64.9633 & 64.9633 & 64.9633 \\
\hline 0 & 11 & 38.7720 & 38.7720 & 38.7720 \\
\hline 0 & 12 & 27.5488 & 27.5488 & 27.5488 \\
\hline 20 & 9 & 154.8534 & 150.4983 & 145.9419 \\
\hline 20 & 11 & 114.5339 & 111.7237 & 108.7090 \\
\hline 20 & 12 & 97.0255 & 94.7439 & 92.2599 \\
\hline 35 & 9 & 140.9664 & 133.1717 & 125.1577 \\
\hline 35 & 11 & 105.9651 & 100.8554 & 95.4591 \\
\hline 35 & 12 & 90.2805 & 86.1055 & 81.6289 \\
\hline 50 & 9 & 124.2946 & 112.9000 & 101.4383 \\
\hline 50 & 11 & 95.3802 & 87.7612 & 79.8703 \\
\hline 50 & 12 & 81.8222 & 75.5473 & 68,9454 \\
\hline
\end{tabular}

* Ocorrência de desbaste aos 4 anos.

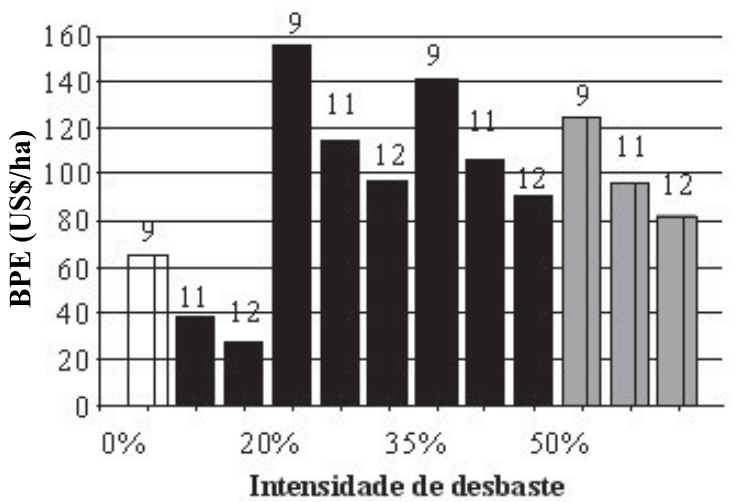

$\square 0 \% \square 20 \% \square 35 \% \square 50 \%$

Figura 3 - Valores de BPE obtidos com desbaste aos 4, 5 e 6 anos nas intensidades de $0,20,35$ e $50 \%$, para índice de local de $25 \mathrm{~m}$, taxa de juros de $8 \%$ e corte final aos 9,11 e 12 anos.

Figure 3 - BPE values obtained with thinning at four, five and six years old in the intensities of $0,20,35$ and $50 \%$, for site index of $25 \mathrm{~m}$, rate of interest of $8 \%$ and last cut at nine, 11 and 12 years.

Scolforo et al. (2001) destacaram que os lucros são sensíveis a variações nas taxas de desconto, ou seja, incrementos na taxa de desconto aumentam o risco do empreendimento, diminuindo a rentabilidade dos regimes de manejo.

\subsection{3. Índice de local}

Consideraram-se, neste caso, uma taxa de juros de $8 \%$ ao ano e a simulação de um desbaste aos 4 anos

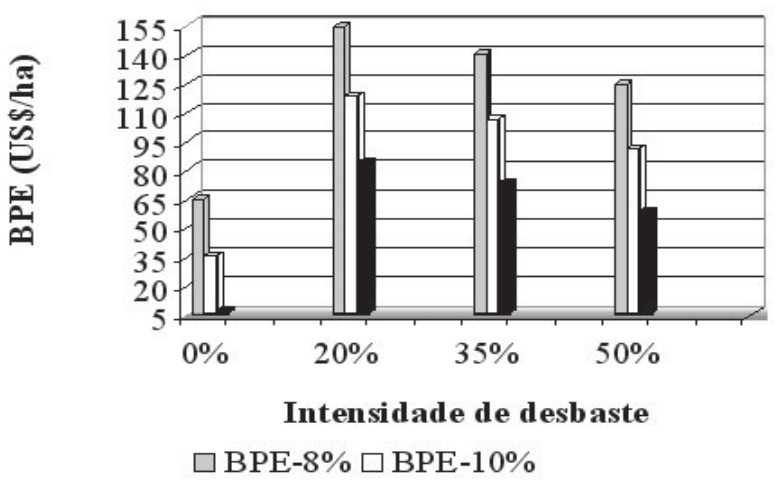

Figura 4 - Valores de BPE para cada intensidade de desbaste, considerando-se taxas de juros de 8,10 e $12 \%$, desbaste aos 4 anos e corte final aos 9 anos.

Figure 4-BPE values per thinning intensity, considering interest rates of 8,10 and $12 \%$, thinning at four years of age and last cut at nine years of age.

com o corte final aos 9 anos, com variações de volume ( $\mathrm{m}^{3} / \mathrm{ha}$ ) de acordo com os índices de local (S) de 17, 21 e $25 \mathrm{~m}$ (Quadro 1). Os valores de BPE apresentaramse maiores em locais mais produtivos, ou seja, com índice de local maior (Figura 5). Esse resultado está de acordo com o esperado do ponto de vista biológico, ou seja, maior índice de local, equivale ao local de maior produtividade, devido a uma maior disponibilidade e melhor utilização pelas árvores dos fatores de crescimento (água, luz e nutrientes), proporcionando, com isso, maior produção volumétrica e, conseqüentemente, maior rendimento econômico. 


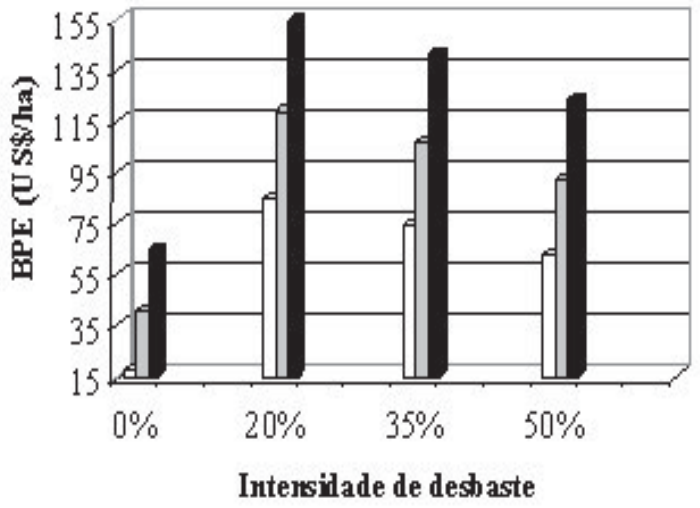

$\square$ site17 $\square$ site21 $\mathbf{Q}_{\text {site } 25}$

Figura 5 -Valores de BPE para cada intensidade de desbaste, considerando-se desbaste aos 4 anos com corte final aos 9 anos e índices de local de 17, 21 e $25 \mathrm{~m}$.

Figure 5-BPE values per thinning intensity, considering thinning at four years of age and last cut at nine yearsof age and site indexes of 17, 21 and $25 \mathrm{~m}$.

\subsection{Efeito das variáveis na determinação da idade de desbaste e corte final}

Nesta etapa, foi analisada a influência de algumas variáveis, como índice de local, taxa de juros, custo de implantação, preço da madeira do desbaste e preço da madeira do corte final, sobre a determinação das idades de desbaste e de corte final mais rentáveis economicamente, avaliados em valores de BPE (US\$.ha-1 $\mathrm{ano}^{-1}$ ).

\subsection{1. Índice de local}

\section{-Idade de desbaste}

Nesta análise, obtiveram-se os valores de BPE de cada regime, considerando a ocorrência de desbaste aos 4, 5 e 6 anos, na intensidade de $35 \%$, taxa de juros de $8 \%$ ao ano e índices de local de 17, 21 e $25 \mathrm{~m}$.

Observou-se que essa variável não influenciou a determinação da idade de desbaste mais rentável, permanecendo a idade de desbaste de 4 anos como o regime que proporcionou maior rendimento para todos os índices de local avaliado e qualquer idade de corte final (Quadro 3).

\section{-Idade de corte final}

Verificou-se que o regime com a idade de corte final de 9 anos foi o que proporcionou maiores rendimentos, medidos em valores de BPE, não sendo influenciado pelos índices de local analisados: 17, 21 ou 25 m (Quadro 3).

\subsubsection{Taxa de juros}

\section{-Idade de desbaste}

Considerou-se neste caso a ocorrência de um desbaste aos 4, 5 e 6 anos, na intensidade de $35 \%$, corte final aos 9, 11 e 12 anos, índice de local de 25 $\mathrm{m}$ e as taxas de juros de 8,10 e $12 \%$ ao ano. Observouse que o regime com a idade de desbaste de 4 anos foi o que forneceu maiores valores de BPE, para qualquer uma das taxas de juros utilizadas (Quadro 4).

Quadro 3 - BPE (US\$.ha- ${ }^{-1} \cdot$ ano $^{-1}$ ) obtidos na simulação de um desbaste de 35\%, aos 4, 5 e 6 anos, taxa de juros de $8 \%$, três níveis de índice de local e diferentes idades de corte final

Table 3 - BPE (in US\$.ha . $^{-1}$ year ${ }^{-1}$ ) obtained in the simulation of a $35 \%$ thinning intensity at four, five and six years of age, interest rate of $8 \%$, three levels of site index and various last cut ages

\begin{tabular}{|c|c|c|c|c|c|}
\hline Desbaste $(\%)$ & Índice de Local & Id. Corte Final & $\begin{array}{c}4-\mathrm{BPE} \\
\left(\mathrm{US} \$ . \mathrm{ha}^{-1} \mathrm{ano}^{-1}\right)\end{array}$ & $\begin{array}{c}5-\mathrm{BPE} \\
\left(\mathrm{US} \$ \cdot \mathrm{ha}^{-1} \mathrm{ano}^{-1}\right)\end{array}$ & $\begin{array}{c}6-\mathrm{BPE} \\
\left(\mathrm{US} \$ . \mathrm{ha}^{-1} \mathrm{ano}^{-1}\right)\end{array}$ \\
\hline & & 9 & 74.5007 & 68.7257 & 62.7770 \\
\hline & & 11 & 51.7026 & 47.9077 & 43.8905 \\
\hline \multirow[t]{2}{*}{35} & 17 & 12 & 41.1156 & 38.0146 & 34.6806 \\
\hline & & 9 & 106.6402 & 99.8799 & 92.9252 \\
\hline \multirow[t]{3}{*}{35} & 21 & 11 & 77.8126 & 73.3779 & 68.6906 \\
\hline & & 12 & 64.7286 & 61.1050 & 57.2161 \\
\hline & & 9 & 140.9664 & 133.1717 & 125.1577 \\
\hline \multirow[t]{2}{*}{35} & 25 & 11 & 105.9651 & 100.8554 & 95.4591 \\
\hline & & 12 & 90.2805 & 86.1055 & 81.6289 \\
\hline
\end{tabular}

R. Árvore, Viçosa-MG, v.29, n.3, p.419-429, 2005 
Quadro 4 - BPE (US\$.ha ${ }^{-1}$. ano $^{-1}$ ) obtidos na simulação de um desbaste de $35 \%$ aos 4,5 e 6 anos, considerando-se as taxas de juros de 8,10 e $12 \%$ e índice de local 25

Table $4-B P E$ (in US\$. $h a^{-1}$.year ${ }^{-1}$ ) obtained from the $35 \%$ thinning intensity simulation at four, five and six years of age, considering the interest rate of 8,10 and $12 \%$, and a site index of $25 \mathrm{~m}$

\begin{tabular}{|c|c|c|c|c|}
\hline Taxa de juros & Corte Final & $\begin{array}{c}4-\mathrm{BPE} \\
\left(\mathrm{US} \$ . \mathrm{ha}^{-1} \mathrm{ano}^{-1}\right)\end{array}$ & $\begin{array}{c}5-\mathrm{BPE} \\
\left(\mathrm{US} \$ \cdot \mathrm{ha}^{-1} \mathrm{ano}^{-1}\right)\end{array}$ & $\begin{array}{c}6-\mathrm{BPE} \\
\left(\mathrm{US} \$ \cdot \mathrm{ha}^{-1} \mathrm{ano}^{-1}\right)\end{array}$ \\
\hline \multirow{3}{*}{$8 \%$} & 9 & 140.9664 & 133.1717 & 125.1577 \\
\hline & 11 & 105.9651 & 100.8554 & 95.4591 \\
\hline & 12 & 90.28054 & 86.1055 & 81.6289 \\
\hline \multirow{3}{*}{$10 \%$} & 9 & 106.1388 & 98.8781 & 91.3577 \\
\hline & 11 & 70.0629 & 65.4185 & 60.4501 \\
\hline & 12 & 54.1685 & 50.4242 & 46.3417 \\
\hline \multirow{3}{*}{$12 \%$} & 9 & 72.6055 & 65.8288 & 58.7621 \\
\hline & 11 & 35.8748 & 31.6374 & 27.0473 \\
\hline & 12 & 19.9695 & 16.5946 & 12.8534 \\
\hline
\end{tabular}

\section{-Idade de corte final}

Observa-se, no Quadro 4, que o regime com a idade de corte final de 9 anos apresentou valores de BPE significativamente superiores em relação aos obtidos com o corte final aos 11 e 12 anos, em qualquer idade de desbaste e taxa de juros.

\subsubsection{Custo de implantação}

\section{- Idade de desbaste}

Foi simulada a ocorrência de desbaste nas idades de 4,5 e 6 anos com uma intensidade de $35 \%$ e idades de corte final de 9, 11 e 12 anos. Consideram-se, neste caso, o índice de local de $25 \mathrm{~m}$, taxa de juros de $8 \%$ ao ano e diferentes custos de implantação. Observouse, no Quadro 5, que o regime com idade de desbaste de 4 anos proporcionou valores de BPE superiores a todos os valores de custo de implantação analisados. À medida que aumentou o custo de implantação, ocorreu diminuição dos valores de BPE para qualquer um dos regimes de manejo analisados.

\section{- Idade de corte final}

O regime mais rentável foi o que considerou a idade de corte final aos 9 anos, apresentando valores de BPE superiores, ao regime com corte final aos 11 e 12 anos, independentemente do custo de implantação analisado (Quadro 5).

\subsubsection{Preço da madeira do desbaste}

\section{- Idade de desbaste}

Para analisar a influência desta variável, optouse por considerar a diferenciação do preço da madeira do desbaste com a idade de colheita, ou seja, para o desbaste aos 4 anos o preço seria de US\$9.00, aos 5 anos US\$11.00 e aos 6 anos US\$13.00 (Figura 2). O índice de local considerado foi de $25 \mathrm{~m}$, a taxa de juros de $8 \%$ e o desbaste de $35 \%$ de retirada em área basal.

Quadro 5 - BPE (US\$.ha ${ }^{-1}$. ano $o^{-1}$ ) para simulação de um desbaste de $35 \%$ aos 4, 5 e 6 anos, índice de local de $25 \mathrm{~m}$ e taxa de juros de $8 \%$, para diferentes custos de implantação

Table 5 - BPE (in US\$.ha $a^{-1}$.year ${ }^{-1}$ ) for a $35 \%$ thinning intensity simulation at four, five and six years of age, site index of $25 \mathrm{~m}$, and interest rate of $8 \%$ for various establishment costs

\begin{tabular}{|c|c|c|c|c|}
\hline $\begin{array}{l}\text { Custo de Implantação } \\
\left(\mathrm{US} \$ \cdot \mathrm{ha}^{-1}\right)\end{array}$ & Corte Final (anos) & $\begin{array}{c}4-\mathrm{BPE} \\
\left(\mathrm{US} \$ \cdot \mathrm{ha}^{-1} \mathrm{ano}^{-1}\right)\end{array}$ & $\begin{array}{c}5-\mathrm{BPE} \\
\left(\mathrm{US} \$ \cdot \mathrm{ha}^{-1} \mathrm{ano}^{-1}\right)\end{array}$ & $\begin{array}{c}6-\mathrm{BPE} \\
\left(\mathrm{US} \$ . \mathrm{ha}^{-1} \mathrm{ano}^{-1}\right)\end{array}$ \\
\hline \multirow{3}{*}{400.00} & 9 & 140.9664 & 133.1717 & 125.1577 \\
\hline & 11 & 105.9651 & 100.8554 & 95.4591 \\
\hline & 12 & 90.2805 & 86.1055 & 81.6289 \\
\hline \multirow{3}{*}{500.00} & 9 & 123.3577 & 115.5629 & 107.5489 \\
\hline & 11 & 90.5567 & 85.4470 & 80.0507 \\
\hline & 12 & 75.6841 & 71.5091 & 67.0325 \\
\hline \multirow{3}{*}{600.00} & 9 & 105.7489 & 97.9541 & 89.9402 \\
\hline & 11 & 75.1483 & 70.0386 & 64.6423 \\
\hline & 12 & 61.0876 & 56.9135 & 52.4360 \\
\hline
\end{tabular}

R. Árvore, Viçosa-MG, v.29, n.3, p.419-429, 2005 
Os resultados indicaram que, quando se utilizou o preço de madeira do desbaste diferenciado pela idade de colheita, os valores de BPE foram maiores, e a idade de desbaste que apresentou melhores retornos financeiros passou de 4 para 6 anos (Quadro 6). Portanto, esta variável influenciou significativamente a determinação da idade de desbaste mais rentável.

\section{- Idade do corte final}

Observou-se que a idade de corte final que proporcionou maiores rendimentos permaneceu sendo aos 9 anos, porém os valores de BPE foram maiores quando houve a diferenciação no preço da madeira do desbaste (Quadro 6).

\subsubsection{Preço da madeira do corte final}

\section{- Idade do desbaste}

Para analisar a influência desta variável na determinação das idades de desbaste e de corte final mais rentável, optou-se por considerar variações no preço da madeira de acordo com a idade de corte final (Figura 2). O índice de local considerado foi de $25 \mathrm{~m}$, a taxa de juros de $8 \%$ e o desbaste de $35 \%$ de retirada em área basal.
Verificou-se que, considerando esse aumento no preço da madeira do corte final, o regime com a idade de desbaste de 4 anos foi o que forneceu maiores valores de BPE, mas os valores de BPE foram maiores quando comparados com as simulações sem diferenciação no preço da madeira do corte final (Quadro 7).

\section{- Idade de corte final}

A elevação no preço da madeira do corte final devido à melhoria na qualidade da madeira ocasionou mudança na idade de corte final mais rentável, passando de 9 para 12 anos e resultando em valores de BPE maiores (Quadro 7).

\subsubsection{Elevação no preço da madeira do desbaste e do corte final}

\section{- Idade do desbaste}

Para analisar a influência desta variável na determinação do regime mais rentável, optou-se por considerar variações tanto no preço da madeira do desbaste quanto na madeira do corte final, como está descrito na Figura 2. O índice de local considerado foi de $25 \mathrm{~m}$, a taxa de juros de $8 \%$ e o desbaste de $35 \%$ de retirada em área basal.

Quadro 6 - BPE (US \$.ha ${ }^{-1}$.ano ${ }^{-1}$ ) obtidos na simulação de um desbaste de 35\% aos 4, 5 e 6 anos, índice de local de 25 $\mathrm{m}$ e taxa de juros de $8 \%$, considerando-se diferentes preços de madeira do desbaste

Table 6 - BPE (in US\$.ha-1.year-1) obtained from the 35\% thinning intensity simulation at four, five and six years of age, site index $25 \mathrm{~m}$ and interest rate of $8 \%$ considering various thinned wood prices

\begin{tabular}{|c|c|c|c|c|}
\hline $\begin{array}{l}\text { Preço da Madeira } \\
\text { Desbaste }\left(\mathrm{US} \$ \mathrm{~m}^{3}\right)\end{array}$ & Corte Final (ano) & $\begin{array}{c}* 4-\mathrm{BPE} \\
\left(\mathrm{US} \$ . \mathrm{ha}^{-1} \mathrm{ano}^{-1}\right) \\
\end{array}$ & $\begin{array}{c}5-\mathrm{BPE} \\
\left(\mathrm{US} \$ \cdot \mathrm{ha}^{-1} \mathrm{ano}^{-1}\right) \\
\end{array}$ & 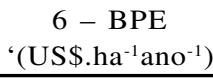 \\
\hline \multirow{3}{*}{9} & 9 & 140.9664 & 133.1717 & 125.1577 \\
\hline & 11 & 105.9651 & 100.8554 & 95.4591 \\
\hline & 12 & 90.2805 & 86.1055 & 81.6289 \\
\hline \multirow{3}{*}{$9,11,13$} & 9 & 140.9664 & 141.1683 & 141.8551 \\
\hline & 11 & 105.9651 & 107.8528 & 110.0700 \\
\hline & 12 & 90.2805 & 92.7342 & 95.4700 \\
\hline
\end{tabular}

Quadro 7 - BPE (US\$.ha-1.ano-1) obtidos na simulação de um desbaste de 35\% aos 4, 5 e 6 anos, índice de local de 25 $\mathrm{m}$ e taxa de juros de $8 \%$ para diferentes preços de madeira do corte final

Table 7 - BPE (in US\$.ha-1.year-1) obtained from the 35\% thinning intensity simulation at four, five and six years of age, site index of $25 \mathrm{~m}$ and interest rate of $8 \%$ for various last cut wood prices

\begin{tabular}{|c|c|c|c|c|}
\hline $\begin{array}{l}\text { Preço da Madeira } \\
(\mathrm{CF})\left(\mathrm{US} \$ / \mathrm{m}^{3}\right)\end{array}$ & Corte Final (ano) & $\begin{array}{c}4-\mathrm{BPE} \\
\left(\mathrm{US} \$ . \mathrm{ha}^{-1} \mathrm{ano}^{-1}\right)\end{array}$ & $\begin{array}{c}5-\mathrm{BPE} \\
\left(\mathrm{US} \$ \cdot \mathrm{ha}^{-1} \mathrm{ano}^{-1}\right)\end{array}$ & $\begin{array}{c}6-\mathrm{BPE} \\
\left(\mathrm{US} \$ \cdot \mathrm{ha}^{-1} \mathrm{ano}^{-1}\right)\end{array}$ \\
\hline & 9 & 140.9664 & 133.1717 & 125.1577 \\
\hline \multirow[t]{2}{*}{25} & 11 & 105.9651 & 100.8554 & 95.4591 \\
\hline & 12 & 90.2805 & 86.1055 & 81.6289 \\
\hline \multirow[t]{3}{*}{$25,30,35$} & 9 & 140.9664 & 133.1717 & 125.1577 \\
\hline & 11 & 160.2215 & 153.5907 & 146.7159 \\
\hline & 12 & 189.0250 & 182.3094 & 128.4932 \\
\hline
\end{tabular}


Verificou-se que, considerando essa elevação nos preços da madeira do desbaste e do corte final, o regime com a idade de desbaste de 6 anos foi o que forneceu maiores valores de BPE, ou seja, postecipou a idade de desbaste mais rentável (Quadro 8).

\section{- Idade de corte final}

As variações nos preços da madeira do desbaste e do corte final influenciaram a determinação do regime mais rentável, ocasionando mudanças na idade de corte final que passou de 9 para 12 anos (Quadro 8).

\subsection{Resumo da influência das variáveis na determinação do melhor regime}

Elaborou-se o Quadro 9 para destacar a amplitude das variáveis avaliadas e o comportamento de cada uma em relação ao valor de BPE e em relação às idades de desbaste e de corte final mais rentáveis economicamente.
No Quadro 9, pode-se observar que, quando se aumenta a intensidade de desbaste, ocorre a diminuição dos valores de BPE, porém a variação nas intensidades não interfere na idade de desbaste e de corte final mais rentáveis, que nesse caso foi de 4 anos e de 9 anos, respectivamente.

Para o índice de local, conforme o esperado, quanto maior o índice, maior o valor de BPE, mudanças nesses índices também não influenciaram as idades de 4 e 9 anos de maior produtividade.

O aumento da taxa de juros acarretou a diminuição dos valores de BPE, sem interferir nas idades de maior rentabilidade. $\mathrm{O}$ mesmo efeito foi observado nos custos de implantação. No entanto, quando se consideraram diferenças no preço da madeira de acordo com a idade do desbaste, houve alteração na idade de desbaste de maior rentabilidade, mudando de 4 para 6 anos, e a idade de corte final permaneceu aos 9 anos como

Quadro 8-BPE (US\$.ha-1.ano-1) obtidos na simulação de um desbaste de 35\% aos 4, 5 e 6 anos, índice de local de 25 $m$ e taxa de juros de $8 \%$, considerando-se diferentes preços de madeira do desbaste e do corte final

Table 8 - BPE (in US\$.ha-1.year-1) obtained from the 35\% thinning intensity simulation at four, five and six years of age, site index $25 \mathrm{~m}$ and interest rate of $8 \%$ considering various prices of thinned and last cut woods

\begin{tabular}{|c|c|c|c|c|c|}
\hline $\begin{array}{l}\text { Preço / Desbaste } \\
\left(\mathrm{US} \$ / \mathrm{m}^{3}\right)\end{array}$ & $\begin{array}{c}\text { Preço/C. Final } \\
\left(\mathrm{US} \$ / \mathrm{m}^{3}\right)\end{array}$ & $\begin{array}{c}\text { C. Final } \\
\text { (anos) }\end{array}$ & $\begin{array}{c}4-\mathrm{BPE} \\
\left(\mathrm{US} \$ \cdot \mathrm{ha}^{-1} \mathrm{ano}^{-1}\right)\end{array}$ & $\begin{array}{c}5-\mathrm{BPE} \\
\left(\mathrm{US} \$ . \mathrm{ha}^{-1} \mathrm{ano}^{-1}\right)\end{array}$ & $\begin{array}{c}6-\mathrm{BPE} \\
\left(\mathrm{US} \$ \cdot \mathrm{ha}^{-1} \mathrm{ano}^{-1}\right)\end{array}$ \\
\hline \multirow{3}{*}{9} & & 9 & 140.9664 & 133.1717 & 125.1577 \\
\hline & 25 & 11 & 105.9651 & 100.8554 & 95.4591 \\
\hline & & 12 & 90.2805 & 86.1055 & 81.6289 \\
\hline \multirow[t]{3}{*}{$9,11,13$} & $25,30,35$ & 9 & 140.9664 & 141.1683 & 141.8551 \\
\hline & & 11 & 160.2215 & 160.5881 & 161.3268 \\
\hline & & 12 & 189.0250 & 188.9380 & 189.1985 \\
\hline
\end{tabular}

Quadro 9- Relação das variáveis com o valor de BPE e a influência na determinação da idade de desbaste e de corte final mais rentável

Table 9 - List of the variables with the BPE value and their influence on the determination of the most profitable thinning and last cut ages

\begin{tabular}{lcccc}
\hline $\begin{array}{c}\text { Amplitude } \\
\text { de Variação }\end{array}$ & Variável & BPE & $\begin{array}{c}\text { Idade de } \\
\text { Desbaste }\end{array}$ & $\begin{array}{c}\text { Idade de } \\
\text { Corte Final }\end{array}$ \\
\hline$(0,20,35,50 \%)$ & \#-Intensidade de desbaste & $\exists$ BPE & $=(4)$ & $=(9)$ \\
$(17,21,25 \mathrm{~m})$ & \#- Índice de local & \# BPE & $=(4)$ & $=(9)$ \\
$(8,10,12 \%)$ & \#-Taxa de juros & $\exists$ BPE & $=(4)$ & $=(9)$ \\
$(400,500,600$ US\$ $/$ ha) & \#-Custo de implantação & $\exists$ BPE & $=(4)$ & $=(9)$ \\
$9,11,13$ US\$ & $\otimes$ no preço da madeira (com a idade do desbaste) & $\#$ BPE & $\otimes(4 \rightarrow 6)$ & $=(9)$ \\
$25,30,35$ US\$ & $\otimes$ no preço da madeira (com a idade de corte final) & $\#$ BPE & $=(4)$ & $\otimes(9 \rightarrow 12)$ \\
$9,11,13$ US\$ & $\otimes$ no preço da madeira & \# BPE & $\otimes(4 \rightarrow 6)$ & $\otimes(9 \rightarrow 12)$ \\
$25,30,35$ US\$ & Desbaste e corte final & & & \\
\hline
\end{tabular}

\# aumento dos valores de BPE; $\exists$ diminui os valores de BPE; = não houve diferenciação com relação ao aumento no valor das variáveis; $\otimes$ diferiu com o aumento no valor das variáveis; $\rightarrow$ mudança de 4 para 6 ou de 9 para 12 .

R. Árvore, Viçosa-MG, v.29, n.3, p.419-429, 2005 
a mais rentável. No caso de variações no preço da madeira do corte final de acordo com a idade, acarretou mudanças na idade de corte final mais rentável, passando de 9 anos para 12 anos.

As mudanças no preço da madeira do desbaste e do corte final, de acordo com a idade, ocorridas de forma simultânea, ocasionaram a postecipação das idades de maior rentabilidade, passando de 4 para 6 anos (desbaste) e de 9 para 12 anos (corte final).

\section{CONCLUSÕES}

O regime de manejo que considerou a ocorrência de desbaste foi mais rentável que o regime sem desbaste, em todas as situações avaliadas. Entretanto, à medida que se aumentou à intensidade de desbaste, houve diminuição dos valores de BPE.

$\mathrm{Na}$ análise dos regimes com desbaste, o regime mais rentável foi com desbaste ocorrendo aos 4 anos e o corte final aos 9 anos, independentemente da intensidade de desbaste, da taxa de juros e do índice de local. Entretanto, maiores valores de taxa de juros ocasionaram diminuição dos valores de BPE em todos os regimes analisados. Entretanto, locais de maior capacidade produtiva tenderam a dar melhores respostas ao regime com desbaste, resultando em maiores valores de BPE.

Não houve influência das variáveis índice de local, taxa de juros e custo de implantação sobre a determinação do regime mais rentável. Em todas as situações simuladas com essas variáveis, o regime mais rentável continuou sendo de desbaste aos 4 e corte final aos 9 anos.

A variação no preço da madeira do desbaste e do corte final com a idade de colheita influenciou a determinação do regime mais rentável. Para a variável preço da madeira do desbaste, o regime mais rentável foi de desbaste aos 6 e corte final aos 9 anos. Para a variável preço da madeira do corte final foi de desbaste aos 4 anos e o corte final aos 12. Quando considerou ao mesmo tempo a elevação dos preços da madeira do desbaste e do corte final, observou-se que o regime mais rentável foi do desbaste aos 6 anos e o corte final aos 12. Além disso, os valores de BPE aumentaram com a elevação do preço da madeira, devido à consideração de melhoria na qualidade da madeira proveniente do povoamento desbastado. As variáveis preço da madeira do desbaste e do corte final influenciaram diretamente a determinação do regime mais rentável.

\section{REFERÊNCIAS BIBLIOGRÁFICAS}

BERTOLOTTI, G. et al. Efeitos de diferentes métodos e intensidades de desbastes na produtividade de Pinus caribaea. var. hondurensis Barr. Et. Golf. Revista IPEF, n.24, p.47-54,1983.

\section{CENTRO DE ESTUDOS AVANÇADOS EM}

ECONOMIA APLICADA - CEPEA (disponível na internet $<$ www.cepea.esalq.br/flore/zip/ inform16.pdf >em abril de 2003).

CLUTTER, J.L. Compatible growth and yield models for loblolly pine. Forest Science, v.9, n.3, p.354-371, 1963.

CLUTTER, J.L. et al. Timber management: a quantitative approach. New York: Wiley, 1983. $333 \mathrm{p}$.

DIAS, A.N. Modelagem e avaliação econômica de plantações de eucalipto submetidas a desbastes. 2000. 70 f. Tese (Mestrado em Ciência Florestal) - Universidade Federal de Viçosa, Viçosa, 2000.

REZENDE, J.L.P.; OLIVEIRA, A.D. Avaliação de projetos florestais. Viçosa, MG: Universidade Federal Viçosa, 1995. 46p.

SCOLFORO, J.R.S. et al. Simulação e avaliação econômica de regimes de desbastes e desrama para obter madeira de Pinus Taeda livre de nós. Ciência Florestal, v.11, n.1, p.121-139, 2001. 